\title{
Positionspapier der DSK zur Datenschutz-Grundverordnung
}

Die Datenschutz-Grundverordnung muss in wesentlichen Punkten nachgebessert werden!

Die Beratungen über die Datenschutz-Grundverordnung sind mit dem Trilog zwischen Europäischem Parlament, Rat der Europäischen Union und Europäischer Kommission in die entscheidende Phase eingetreten. Für die Konferenz der Datenschutzbeauftragten des Bundes und der Länder ist es von außerordentlicher Bedeutung, dass die Datenschutz-Grundverordnung im Vergleich zum geltenden Rechtsstand einen verbesserten, mindestens aber dem bisherigen Standard gleichwertigen Grundrechtsschutz gewährleistet. Sie appelliert an die Trilogpartner, bei ihren Verhandlungen insbesondere zu berücksichtigen:

\section{Die Datensparsamkeit muss Gestaltungsziel bleiben!}

Die Allgegenwärtigkeit der Datenverarbeitung und der Einsatz von Big-Data-Technologien erzeugen eine unvorstellbare Menge (auch personenbezogener) Daten. Deshalb ist das seit vielen Jahren im deutschen Datenschutzrecht verankerte Prinzip der Datenvermeidung und Datensparsamkeit wichtiger denn je. Für eine möglichst grundrechtsschonende Datenverarbeitung müssen sich sowohl Staat als auch Wirtschaft auf das zur Erreichung ihrer im Einklang mit der Rechtsordnung legitimen Zwecke notwendige Maß beschränken. Das Prinzip der Datensparsamkeit muss durch die Datenschutz-Grundverordnung explizit vorgegeben werden.

\section{Es darf keine Aufweichung der Zweckbindung geben!}

Der Grundsatz der Zweckbindung dient in erster Linie der Transparenz und Vorhersehbarkeit der Datenverarbeitung und stärkt die Autonomie der Betroffenen, indem sie sich darauf verlassen können, dass ihre Daten nur zu Zwecken weiterverarbeitet werden, zu denen sie erhoben wurden. Insbesondere durch die vom Rat vorgeschlagene Regelung würden Zweckänderungen in einem derart weiten Umfang zulässig, dass das in der Europäischen Grundrechtecharta enthaltene Prinzip der Zweckbindung, preisgegeben wäre. Dies lehnt die Konferenz entschieden ab.

Auch die vom Rat vorgesehenen Privilegierungen für die Datenverarbeitung zu statistischen, historischen und wissenschaftlichen Zwecken, nach denen vom ursprünglichen Erhebungszweck abweichende Verarbeitungen stets nahezu schrankenlos zulässig sind, begegnen erheblichen Bedenken.

\section{Die Einwilligung des Einzelnen muss die Datenhoheit si- chern!}

Recht auf informationelle Selbstbestimmung bedeutet, dass der Einzelne grundsätzlich selbst über die Preisgabe und Verwendung seiner personenbezogenen Daten in der Form der Einwilligung entscheiden kann. Die Einwilligung ist aber nur dann ein wesentliches Element zur Gewährleistung der Datenhoheit, wenn sie durch eine ausdrückliche Willensbekundung erfolgt. Einwilligungserklärungen, die - wie der Rat vorschlägt - lediglich unmissverständlich sein müssen, lehnt die Konferenz als unzureichend ab. Letzteres ermöglicht es den global agierenden Diensteanbietern, durch die Verwendung pauschaler Datenschutzbestimmungen und datenschutzunfreundlicher Voreinstellungen weitreichende Datenverarbeitungsbefugnisse ohne ausdrückliche Einwilligung des Nutzers für sich zu reklamieren. Damit wird einem Opt-out als pauschale Möglichkeit der Einwilligung der Weg bereitet.

\section{Die Rechte der Betroffenen dürfen nicht eingeschränkt wer- den!}

Die Konferenz spricht sich für umfassende Informationsrechte aus, die die Betroffenen in die Lage versetzen, Umfang und Risiko der Datenverarbeitung einzuschätzen. Die Ausübung ihrer Rechte und die zur Umsetzung ergriffenen Maßnahmen müssen für die Betroffenen unentgeltlich sein. Die Konferenz wendet sich daher gegen die vom Rat vorgesehenen diesbezüglichen Beschränkungen aus.

\section{Die Profilbildung muss wirksam begrenzt werden!}

Die Konferenz weist erneut auf die Notwendigkeit einer strikten Regelung der Profil-bildung hin, die der Zusammenführung und Auswertung personenbezogener Daten über eine Person enge Grenzen setzt. Die vorgesehenen Regelungen greifen hier zu kurz.

6. Effektiver Datenschutz braucht betriebliche und behördliche Datenschutzbeauftragte!

Für die Effektivität der Datenschutzaufsicht kommt den in Deutschland fest etablierten behördlichen und betrieblichen Datenschutzbeauftragten große Bedeutung zu. Die Konferenz setzt sich dafür ein, dass die Bestellung von Datenschutzbeauftragten in Behörden und Unternehmen europaweit verpflichtend ist.

7. Datenübermittlungen an Behörden und Gerichte in Drittstaaten bedürfen einer stärkeren Kontrolle!

Nach den Datenschutzskandalen der jüngsten Zeit ist ein besserer Schutz der personenbezogenen Daten von europäischen Bürgerinnen und Bürgern gegenüber drittstaatlichen Einrichtungen dringend geboten. Nach dem Vorschlag des Parlamentes sollen Urteile von Gerichten und Entscheidungen von Verwaltungsbehörden eines Drittstaates, die von einer datenverarbeitenden Stelle die Weitergabe personenbezogener Daten verlangen, in der EU nur auf der Grundlage internationaler Übereinkommen zur Amts- und Rechtshilfe anerkannt und vollstreckt werden. 